\title{
Vaccination Against Cervical Cancer in India: Our Children Deserve a Healthier Future
}

\author{
Venkatraman Radhakrishnan ${ }^{1, \odot ~}$ Catherine G Lam² \\ 1Department of Medical and Pediatric Oncology, Cancer Institute \\ (WIA), Adyar, Chennai, Tamil Nadu, India \\ 2Director of Health Systems and Asia Pacific Regional Programs, St. \\ Jude Children's Research Hospital, Memphis, TN, USA. \\ 3 Professor and Associate Dean for Research in the College of \\ Nursing University of Tennessee College of Health Sciences, \\ Memphis, TN, USA
}

Ind J Med Paediatr Oncol 2021;42:190-193.

\section{Executive Summary}

- Cancer of the uterine cervix is the second most common cancer after breast cancer among Indian women.

- About 604,127 women get this disease every year in the world and $56 \%$ of them die.

- One woman in India dies every 8 minutes from cervical cancer.

- It mainly affects young women belonging to the lower socioeconomic strata in the productive years of their lives (21-67 years of age).

- The human papilloma virus (HPV) is responsible for the majority of cervical cancers and an effective vaccine is available against HPV.

- Vaccination is most effective when given to young girls between the ages of 9 to 13 years before they become sexually active.

- There is adequate evidence in the literature on the cost-effectiveness of HPV vaccination in preventing cervical cancer.

\section{Recommendations}

- HPV vaccination should be incorporated into the national immunization schedule.

- It should be provided free of cost by the government to all females aged between 9 to 13 years.

\section{Introduction}

Cancer of the uterine cervix is the second most common cancer after breast cancer in Indian women, and it is the third most common cancer among all cancers across both

DOI https://doi.org/ $10.1055 / \mathrm{s}-0041-1731843$ ISSN 0971-5851
Address for correspondence Venkatraman Radhakrishnan, MD, DM, Medical and Pediatric Oncology, Cancer Institute (WIA), Adyar, Chennai, Tamil Nadu, 600078, India (e-mail: venkymd@gmail.com).

genders. ${ }^{1}$ In India, it is one of the leading causes of death due to cancer. ${ }^{2}$ It impacts women during the most productive years of their lives.

GLOBOCAN reported that in 2020, 604,127 women were diagnosed with cervical cancer and 341, 831 women died due to cervical cancer across the world. ${ }^{1}$ That is approximately $56 \%$ of women diagnosed with cervical cancer will succumb to the disease. ${ }^{1}$ As per the GLOBOCAN data, 123,907 , cervical cancer cases were diagnosed in India in 2020 and 77,348 patients died due to the disease. India accounts for $20 \%$ of newly diagnosed cervical cancer cases and $22 \%$ of deaths due to cervical cancer in the world every year. ${ }^{1}$

The median age for diagnosis of cervical cancer is 38 years. ${ }^{3}$ The majority of sexually active women $(80 \%)$ will be infected with HPV by the time they are 50 years old. ${ }^{4}$

HPV is the causative agent for most cervical cancers and other factors like infection with human immunodeficiency virus (HIV), genital Chlamydia trachomatis infection, multiple childbirths, smoking, and long-term oral contraceptive use increase the risk of HPV-induced cervical cancer. ${ }^{1}$

HPV strains 16 and 18 contribute to 80 to $85 \%$ of cases in India. ${ }^{2}$ Clinical trials have shown a high degree of efficacy for the HPV vaccine in preventing cervical intraepithelial neoplasia (CIN) and cervical cancer. ${ }^{5,6}$

\section{Methods}

A literature search was done on PUBMED and Google for articles on the safety, efficacy, and cost-effectiveness of HPV vaccination in preventing cervical cancer or the premalignant condition CIN. Articles on the above subject published between 2005 and 2020 were reviewed to synthesize the evidence. (c) 2021. Indian Society of Medical and Paediatric Oncology.

This is an open access article published by Thieme under the terms of the Creative Commons Attribution-NonDerivative-NonCommercial-License, permitting copying and reproduction so long as the original work is given appropriate credit. Contents may not be used for commercial purposes, or adapted, remixed, transformed or built upon. (https://creativecommons.org/licenses/by-nc-nd/4.0/).

Thieme Medical and Scientific Publishers Private Ltd. A-12, Second Floor, Sector -2, NOIDA -201301, India 


\section{Why is Vaccination the Best Prevention?}

The options available to decrease the incidence of cervical cancer are lifetime sexual abstinence, monogamy with a monogamous partner, or HPV vaccination. Lifetime sexual abstinence and monogamy are not practical solutions; therefore, HPV vaccination is the most important tool in reducing the incidence of cervical cancer. HPV vaccination should ideally be administered to girls between the ages of 9 to 13 years before they become sexually active..$^{25,7}$

\section{Vaccination is Beneficial, Safe, and Cost-Effective}

The Papillomavirus Rapid Interface for Modelling and Economics (PRIME) study looked at the cost-effectiveness of vaccinating females against HPV before initiation of sexual life in 179 countries. The study reported that if HPV vaccination is introduced in countries where it is not available then it is estimated that by 2070 , there will be a reduction in $4,00,000$ cases and $2,00,000$ deaths every year due to cervical cancer. The study observed that HPV vaccination is economical. ${ }^{8}$ The biggest beneficiary of this would be India. ${ }^{8,9}$ Furthermore, the benefits of the HPV vaccine extend beyond protection against cervical cancer ( - Table $\mathbf{1}$ ).

The three HPV vaccines approved for use by the US Food and Drug Administration (FDA) are as follows: Gardasil 9 (Merck Frosst Ltd), Gardasil (Merck Frosst Ltd), and Cervarix (GlaxoSmithKline). Gardasil 9 is a nine-valent vaccine that covers HPV strains $6,11,16,18,31,33,45,52$, and 58; Gardasil is a quadrivalent recombinant vaccine that covers HPV-6, 11, 16, and 18 strains; and Cervarix is a bivalent vaccine that covers HPV-16 and 18 strains. HPV strains 6 and 11 cause genital warts. Gardasil and Cervarix are currently available in India. The recommended dose of the HPV vaccine for children aged between 9 to 14 years is two doses ( $0,6-12$ months). In women aged between 15 to 45 years, the recommended dose is three $(0,1-2,6$ months $) .{ }^{12}$ Any of the three approved vaccines can be used to complete the recommended immunization schedule.

A meta-analysis has shown that the immunity provided by two doses of HPV vaccine is sustained for a long time. ${ }^{13} \mathrm{~A}$ Cochrane meta-analysis has shown that the HPV vaccine is most efficacious in young girls, and its efficacy reduces as the age increases due to exposure to HPV. ${ }^{14}$ Immunizing children less than 14 years is not only more protective against cervical cancer but also cost-effective, as only two doses instead of three are required. ${ }^{2,15}$

Table 1 Benefits of vaccination beyond cervical cancer ${ }^{10,11}$

- HPV causes anal cancer, head, and neck SCC, penile cancer, vulva, and vaginal cancer, and anogenital warts.

- Head and neck SCC is the second most common cancer in India (200,000 cases/year) and the most common cancer among men.

- $30-50 \%$ of head and neck SCC in India are HPV positive (higher in the oral cavity).

- Vaccinating females might reduce HPV-related head and neck cancer and anal cancer in men and women through herd immunity. ${ }^{11}$

Abbreviations: HPV, human papilloma virus; SCC, squamous cell carcinoma.
There are multiple studies and meta-analyses that have reported that HPV vaccination is safe and the majority of the adverse events are transient and is due to injection site symptoms like pain, swelling, and redness. ${ }^{14,16}$

\section{Adoption of HPV Vaccine in India}

The government of India in 2008 approved the use of the HPV vaccine in India for the prevention of cervical cancer, and universal HPV vaccination of girls in India has been recommended by the National Technical Advisory Group on Immunization. ${ }^{17} \mathrm{HPV}$ vaccination of all females between the ages of 9 to 14 years was introduced by the state of Sikkim in 2019. ${ }^{2}$ Delhi state has also started a program of opportunistic HPV vaccination for females between the ages of 12 to 13 years. ${ }^{2}$ Punjab state has introduced universal HPV vaccination in two districts for girls aged between 11 to 12 years. $^{2}$ Approximately 40,000 women have been vaccinated with the HPV vaccine in Sikkim, Delhi, and Punjab as mentioned above, and no vaccine-related serious adverse events have been reported from these three states. ${ }^{2}$

\section{Cervical Cancer Screening}

Screening for cervical cancer detects CIN, which is a premalignant lesion. Screening for cervical cancer is effective in reducing morbidity and mortality, because it takes approximately 10 to 20 years for CIN to progress to invasive cancer. ${ }^{18}$ The common screening methods used for cervical cancer include: 1 . Visual inspection with acetic acid or Lugol's iodine (VIA/VILI); 2. Papanicolaou test (Pap test or Pap smear); and 3. HPV deoxyribonucleic acid (DNA) testing. ${ }^{18}$ Limited infrastructure, implementation cost, and lack of trained staff are challenges in implementing universal cervical cancer screening in India. ${ }^{19}$ Most cervical cancer screening in India is opportunistic or done in symptomatic patients. ${ }^{18}$

The American Cancer Society guidelines for screening for cervical cancer recommend that screening should begin at the age of 25 years and continue till the age of 65 years, and HPV DNA testing every 5 years is the preferred option. ${ }^{1}$ The World Health Organization (WHO) recommends VIA or Pap smear-based screening once in 3 to 5 years or DNA-based testing every 5 years for women between the ages of 30 and 49 years in resource-limited settings combined with effective treatment of the precancerous lesion. ${ }^{1}$ The Government of India recommends screening of women aged between 30 to 65 years with VIA once every 5 years. ${ }^{20}$ In India, screening with VIA every 5 years is the most cost-effective compared with Pap smear and HPV DNA testing. ${ }^{21}$

Despite the availability of highly effective vaccines against HPV, the screening programs for cervical cancer should continue to protect women who are not vaccinated and detect cancers caused by HPV strains not covered by the vaccines.

\section{Barriers to Cervical Cancer Screening in India}

Studies have observed a lack of awareness about screening, symptoms, HPV vaccination, and benefits of early detection and treatment of cervical cancer among women in India, especially in rural areas. ${ }^{19}$ Restricted access to health care 
facilities for women for screening and treatment of cervical cancer due to social, economic, and cultural issues and fear of the disease compounds the ignorance associated with the disease. ${ }^{19}$ Older age, illiteracy, lower socioeconomic status, and no history of cancer in the family are associated with decreased awareness of cervical cancer. ${ }^{19}$ Using health communication strategies targeted at high-risk communities has been shown to improve awareness of cervical cancer screening and treatment. ${ }^{19} \mathrm{~A}$ self-administered HPV DNA screening test can also help in overcoming the barriers associated with cervical cancer screening. ${ }^{1}$

\section{Challenges to Implementation of HPV Vaccine in India and Solutions}

Free universal HPV vaccination in India, as a part of the national immunization program, has been delayed due to the high cost of implementation. The market price of a single dose of cervical cancer vaccine is 25 to 40 US dollars (USD) (2,000-3,000 Indian National Rupee [INR]) and for HPV DNA screening test is 13 USD (1,000 INR). At the current market price, the cost of two doses of vaccine and two screening tests is approximately 80 to 106 USD (6,000-8,000 INR). The Global Alliance for Vaccine Initiative (GAVI) price for HPV vaccine is 4.5 USD/dose (293 INR), which is considerably cheaper than the market price. ${ }^{22}$

A study from India in 2008 reported that vaccinating girls (below 12 years of age) with the HPV vaccine and providing two-lifetime cervical cancer screening tests (before 30 years of age) to them would be cost-effective if it was achieved for 10 US dollars (750 INR). ${ }^{23}$ Doing so will lead to a mean risk reduction of $63 \%$ against cervical cancer in the population. The cost of the HPV vaccine and HPV DNA screening test will come down dramatically if the procurement is made by the government and the demand for the vaccine and screening tests increase.

A cost-effectiveness analysis study on vaccinating girls in the state of Punjab against HPV found it to be cost-effective. ${ }^{22}$ We can concur from the Punjab experience that the implementation of the HPV vaccination program in other states will also be cost-effective ${ }^{22}$ ( - Table 2). A recent meta-analysis reported that the HPV vaccine program was economical in resource-constrained settings. ${ }^{24}$

Table 2 Punjab state HPV vaccination cost-benefit analysis study highlights 22

- The net cost of vaccinating 11-year-old girls in the state (1-year cohort) was 135 million INR (2.08 million USD).

- The net cost of vaccination after accounting for the money saved from the reduction of cervical cancer and consequently treatment was 38 million INR (0.58 million USD).

- Vaccination continued to remain cost-effective even if the burden of cervical cancer was reduced by half and the cost of vaccine increased by 2.7 times.

- The vaccination strategy will be $90 \%$ effective at a willingness to pay threshold of 10,000 INR by the consumer. This is less than one-tenth of the per capita GDP.

- For every 1,000 girls who are immunized, three to four cases of cervical cancer will be prevented.

Abbreviations: GDP, gross domestic product; INR, Indian national rupee; USD, US dollar.
There was a media outcry in 2012 after a few deaths were reported in a cervical cancer trial that was conducted in India. ${ }^{2,25,26}$ This led to an adverse public opinion in India against the HPV vaccine.,25,26 The Supreme Court of India also intervened and stopped further cervical cancer vaccine trials in India and questioned the ethical conduct of the trials. ${ }^{2}$ Investigations conducted later found that the deaths in the trials were unrelated to the vaccine. ${ }^{25,26}$ This negative perception of the HPV vaccine in the community needs to be allayed to increase the acceptance of the vaccine.

\section{The Argument Against Universal HPV Vaccination}

Cervical cancer rates have dramatically declined in India in the last few decades. Some states are reporting fewer than 10 cases per 1,00,000 women per year. ${ }^{2}$ This declining trend is cited as an argument against the HPV vaccine, as even without the vaccine, the rates are reducing; therefore, the cost-effectiveness of the vaccine is being questioned and more emphasis on screening is being favored. ${ }^{27}$ The decreasing incidence of cervical cancer in India presents us with the opportunity to eradicate cervical cancer using a combination of vaccination and judicious screening. A declining incidence of cervical cancer should not dissuade us from pursuing universal HPV vaccine as China, which reported a decline in cervical cancer incidence in the past, is observing a rapid rise in the incidence recently. ${ }^{2}$

\section{The Future}

A single dose HPV vaccine will be more cost-effective than two doses of the vaccine. The safety and efficacy of this are being tested in ongoing trials. ${ }^{28}$ Manufacturing the HPV vaccine in India rather than importing the vaccine will also reduce the cost and improve the supply chain. The Serum Institute of India (Pune, India) has manufactured an indigenous quadrivalent HPV vaccine and is currently conducting clinical trials to assess its efficacy and safety. Getting the policies and infrastructure in place now will allow the new vaccines to be feasibly implemented as soon as they are available. There are currently no sensitive or specific tumor markers available for cervical cancer detection. A liquid biopsy-based screening tool will be an attractive option for early detection of cervical cancer, and there is promising data with serum microribonucleic acid (miRNA) for detection and monitoring response to treatment in cervical cancer. ${ }^{29}$

\section{Conclusion}

India was a pioneer in smallpox and polio eradication. We can replicate our past successes with the HPV vaccine for the eradication of cervical cancer. Let us allow our children to lead a healthy future free of cervical cancer.

Childhood cancers cannot be prevented. However, actions one can take during childhood that reduce adult cancers include hepatitis B virus, hepatitis C and HPV vaccination, preventing children from taking up tobacco and alcohol, and reducing obesity, since exposure to these during childhood increases the risk of various adult cancers. 


\section{Conflict of Interest}

None declared.

\section{References}

1 Sung H, Ferlay J, Siegel RL, et al. Global cancer statistics 2020: GLOBOCAN estimates of incidence and mortality worldwide for 36 cancers in 185 countries. CA Cancer J Clin 2021;e-pub ahead of print). doi: 10.3322/caac.21660

2 Sankaranarayanan R, Basu P, Kaur P, et al. Current status of human papillomavirus vaccination in India's cervical cancer prevention efforts. Lancet Oncol 2019;20(11):e637-e644

3 Chatterjee S, Chattopadhyay A, Samanta L, Panigrahi P. HPV and cervical cancer epidemiology - Current status of HPV vaccination in India. Asian Pac J Cancer Prev 2016;17(8):3663-3673

4 Ault KA. Epidemiology and natural history of human papillomavirus infections in the female genital tract. Infect Dis Obstet Gynecol 2006;2006(Suppl):40470

5 Mehrotra R, Hariprasad R, Rajaraman P, et al. Stemming the wave of cervical cancer: human papillomavirus vaccine introduction in India. J Glob Oncol 2018;4:1-4

6 LeiJ, Ploner A, Elfström KM, et al. HPV vaccination and the risk of invasive cervical cancer. N Engl J Med 2020;383(14):1340-1348

7 Kaur P, Mehrotra R, Rengaswamy S, et al. Human papillomavirus vaccine for cancer cervix prevention: rationale \& recommendations for implementation in India. Indian $\mathrm{J}$ Med Res 2017;146(2):153-157

8 Jit M, Brisson M, Portnoy A, Hutubessy R. Cost-effectiveness of female human papillomavirus vaccination in 179 countries: a PRIME modelling study. Lancet Glob Health 2014;2(7):e406-e414

9 Abbas KM, van Zandvoort K, Brisson M, Jit M. Effects of updated demography, disability weights, and cervical cancer burden on estimates of human papillomavirus vaccination impact at the global, regional, and national levels: a PRIME modelling study. Lancet Glob Health 2020;8(4):e536-e544

10 Murthy V, Calcuttawala A, Chadha K, et al. Human papillomavirus in head and neck cancer in India: Current status and consensus recommendations. South Asian J Cancer 2017;6(3):93-98

11 Ong KJ, Checchi M, Burns L, Pavitt C, Postma MJ, Jit M. Systematic review and evidence synthesis of non-cervical human papillomavirus-related disease health system costs and quality of life estimates. Sex Transm Infect 2019;95(1):28-35

12 Center for Disease Control USA guidelines for HPV vaccination. Available at: https://www.cdc.gov/vaccines/vpd/hpv/hcp/ administration.html. Accessed April 2, 2020

13 Deleré Y, Wichmann O, Klug SJ, et al. The efficacy and duration of vaccine protection against human papillomavirus: a systematic review and meta-analysis. Dtsch Arztebl Int 2014;111(35-36):584-591

14 Arbyn M, Xu L. Efficacy and safety of prophylactic HPV vaccines. A Cochrane review of randomized trials. Expert Rev Vaccines 2018;17(12):1085-1091
15 Drolet M, Bénard É, Pérez N, Brisson M; HPV Vaccination Impact Study Group. Population-level impact and herd effects following the introduction of human papillomavirus vaccination programmes: updated systematic review and meta-analysis. Lancet 2019;394(10197):497-509

16 Ogawa Y, Takei H, Ogawa R, Mihara K.. Safety of human papillomavirus vaccines in healthy young women: a meta-analysis of 24 controlled studies. J Pharm Health Care Sci 2017;3-18

17 Senapathy JG, Umadevi P, Kannika PS. The present scenario of cervical cancer control and HPV epidemiology in India: an outline. Asian Pac J Cancer Prev 2011;12(5):1107-1115

18 Krishnamurthy A, Ramshankar V. Current status and future perspectives of molecular prevention strategies for cervical cancers. Indian J Surg Oncol 2020;11(4):752-761

19 Srivastava AN, Misra JS, Srivastava S, Das BC, Gupta S. Cervical cancer screening in rural India: Status \& current concepts. Indian J Med Res 2018;148(6):687-696

20 Operational Framework, Management of Common Cancers. Ministry of Health and Family Welfare, Government of India. Available at: http://cancerindia.org.in/wp-content/ uploads/2017/11/Operational_Framework_Management_of_ Common_Cancers.pdf. Accessed March 18, 2021

21 Chauhan AS, Prinja S, Srinivasan R, et al. Cost effectiveness of strategies for cervical cancer prevention in India. PLoS One 2020;15(9):e0238291

22 Prinja S, Bahuguna P, Faujdar DS, et al. Cost-effectiveness of human papillomavirus vaccination for adolescent girls in Punjab state: Implications for India's universal immunization program. Cancer 2017;123(17):3253-3260

23 Diaz M, Kim JJ, Albero G, et al. Health and economic impact of HPV 16 and 18 vaccination and cervical cancer screening in India. Br J Cancer 2008;99(2):230-238

24 Silas OA, Achenbach CJ, Murphy RL, et al. Cost effectiveness of human papilloma virus vaccination in low and middle income countries: a systematic review of literature. Expert Rev Vaccines 2018;17(1):91-98

25 Das M. Cervical cancer vaccine controversy in India. Lancet Oncol 2018;19(2):e84

26 Cervical vaccine controversy in India. Available at: https:// scroll.in/pulse/865284/efficacy-safety-cost-indias-decadeold-debate-on-the-cervical-cancer-vaccine-erupts-again. Accessed May 2, 2020

27 Gupta S, Kerkar RA, Dikshit R, Badwe RA. Is human papillomavirus vaccination likely to be a useful strategy in India? South Asian J Cancer 2013;2(4):193-197

28 Burger EA, Campos NG, Sy S, Regan C, Kim JJ. Health and economic benefits of single-dose HPV vaccination in a Gavi-eligible country. Vaccine 2018;36(32 Pt A) :4823-4829

29 Laengsri V, Kerdpin U, Plabplueng C, Treeratanapiboon L, Nuchnoi P. Cervical cancer markers: epigenetics and microRNAs. Lab Med 2018;49(2):97-111 